\title{
POLAR measurements of the Crab pulsar
}

\author{
Hancheng $\mathbf{L i}{ }^{a b}$, Mingyu $\mathbf{G e}^{* b}$, Bobing Wu ${ }^{b}$ on behalf of the POLAR collaboration ${ }^{\dagger}$ \\ ${ }^{a}$ University of Chinese Academy of Sciences, Beijing 100049, China \\ ${ }^{b}$ Key Laboratory for Particle Astrophysics, Institute of High Energy Physics, Beijing 100049, \\ China \\ E-mail: gemy@mail.ihep.ac.cn
}

POLAR is a Compton polarimeter sensitive in the 50 to $500 \mathrm{keV}$ energy range. The Crab pulsar is a scientific target for POLAR on board the Chinese space laboratory Tiangong-2(TG-2). With its large Field of View (FoV), POLAR detected significant pulsed signals from the Crab pulsar which is visible by POLAR in about half of all observation time. In this work, we present the preliminary results including the pulse profile, timing and polarization measuring method. First, we show the highly significant pulse profile observed by POLAR which is compared to the results of other detectors including Fermi/LAT and INTEGRAL. The pulse profile as a function of theta incident angle and as a function of channel number, which indicate that POLAR has a good detection performance, have also been showed. Second, we find that the timing of the Crab pulses are accurately measured, which provides a unique verification and calibration to the POLAR timing system. Finally, the potential polarization measurement of the Crab pulsar is also discussed.

35th International Cosmic Ray Conference - ICRC2017

10-20 July, 2017

Bexco, Busan, Korea

\footnotetext{
*Speaker.

${ }^{\dagger}$ Abous us at our website: polar.ihep.ac.cn
} 


\section{Introduction}

The Crab pulsar is one of the most widely studied celestial objects which was born in 1054, has a spin period of $33 \mathrm{~ms}$, with a bright feature over the full electromagnetic spectrum from radio to high energy $\gamma$-rays. At all wavelengths this pulsar shows a double-pulse structure, with the main pulse (P1) and the inter pulse (P2) separated by a phase of $\sim 144^{\circ}$ [1] [2] [3] [4] [5]. In addition, the polarization of the Crab pulsar and Nebulae are also concerned since they have highly magnetized filed. A sounding rocket mission [6] reported the first detection of X-ray polarisation (5-20 keV) for the Crab nebula with a sounding rocket payload. Then, the polarisation fraction was measured with $19.2 \pm 1.0$ ) at $2.6 \mathrm{keV}$ by an instrument on-board the OSO-8 satellite mission [7] during a 256 ks observation. More recently, the INTEGRAL satellite [8] [9], AstroSAT and PoGO+ [10] instruments have also measured the polarisation of Crab emissions.

POLAR started its mission on-board the Chinese space laboratory TG-2 after the successful launch which was on 15th September, 2016 [11]. The installation diagram of POLAR on TG-2 ${ }^{1}$ is shown in Figure 1. POLAR has a high pportunity to capture photons from Crab pulsar thanks to its high sensitivity and large FoV when flying in-orbit, even if POLAR has no pointing control system by itself. The structure of POLAR detector [12] is shown in Figure 2 (a). When photons incident on POLAR, they tend to scatter in the detector. The deposited energy of these scattering photons will be converted to digital signal, and if the signal value exceed certain thresholds, through an complex process by electronic system [13], these trigger informations will be recorded.

The polarisation of Crab pulsed emission can be measured by POLAR since it is a polarimeter especially for GRB with an energy range from 50 to $500 \mathrm{keV}$. The basic concept is to measure the mean degree of polarization and the azimuthal angle of the polarization vector by analyzing the angular distribution of the Compton scattering azimuthal angle of a sample of photons from GRB or other X-ray/ Gamma-ray emissions. The emission of the Crab pulsar can also be observed because the pulsed photon can be accumulated with the steady pulse phase considering the frequency evolution, though it is very faint compared with the GRB flux in a short time interval. However, it is very difficult to acquire the polarization directly because the incident angle between the Crab and POLAR varies with time and the signals could not be accumulated directly. Considering this situation, some new methods should be developed together with the Monte Carlo simulation.

\section{Observations and Data Reduction}

\subsection{Observations}

The Crab observation period for POLAR can obtained only through the judgement of IN or OUT FoV. So we can calculate the incident angle from Crab to POLAR detector by use of platform parameter data(PPD) which is provided in every second. And we can get an exposure map of Crab observation by accumulating the same incident angle of observation together, as shown in Figure 2 (b). Above all, in scientific observation data(SCI), for each event, it has a lot of useful information, such as trigger time, deposited energy signal, trigger position, trigger number, etc. These information support us to perform the following analysis.

\footnotetext{
${ }^{1}$ http://www.collectspace.com/images/news-091516d-lg.jpg
} 


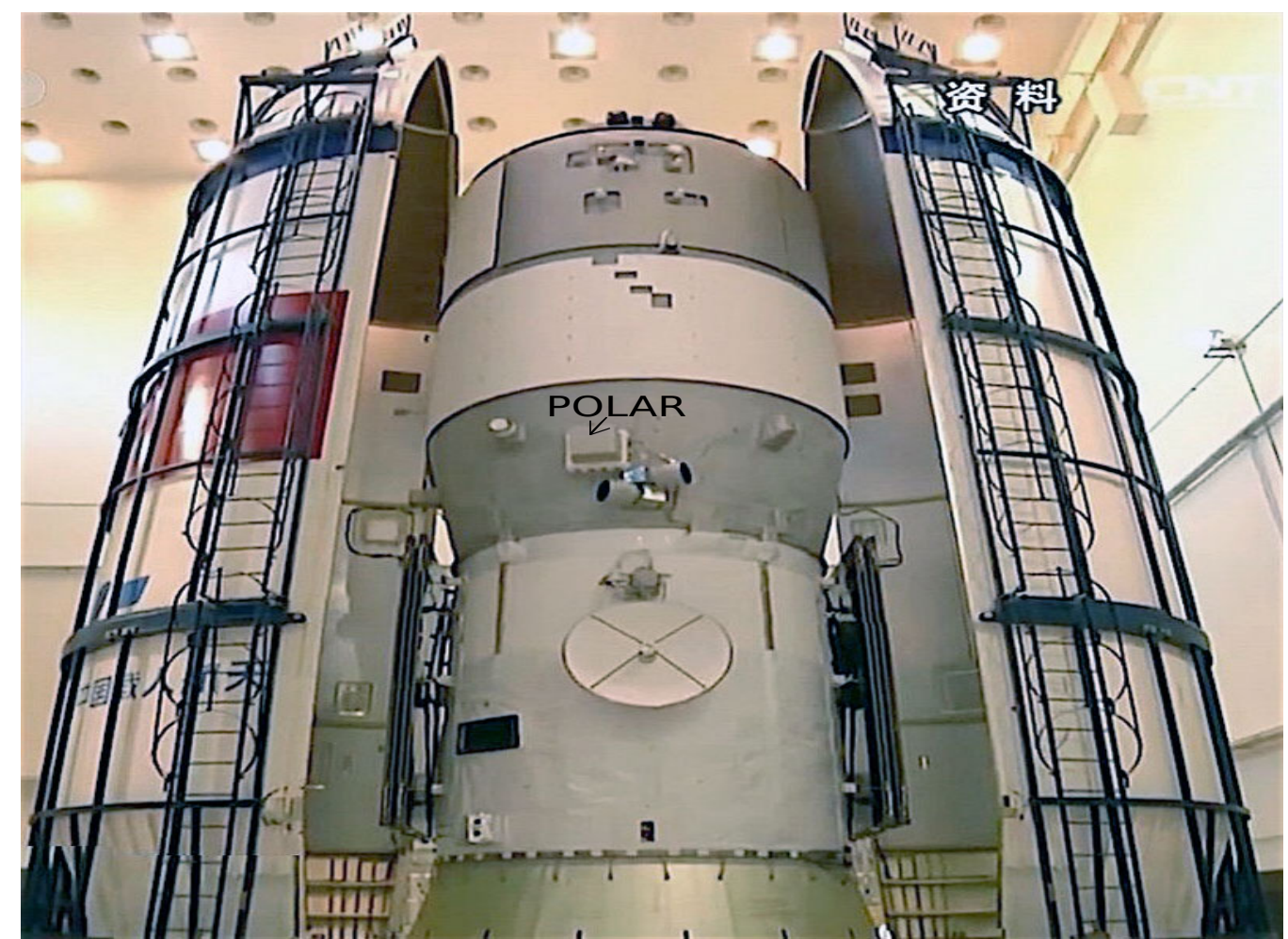

Figure 1: The installation diagram of POLAR on TG-2. The outboard part is called obox, it's connected with ibox which is supporting electronics system inboard.

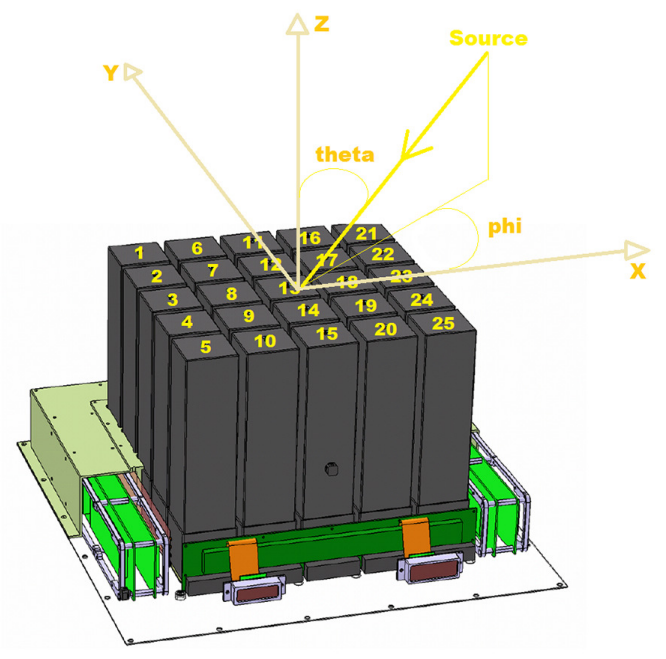

(a)

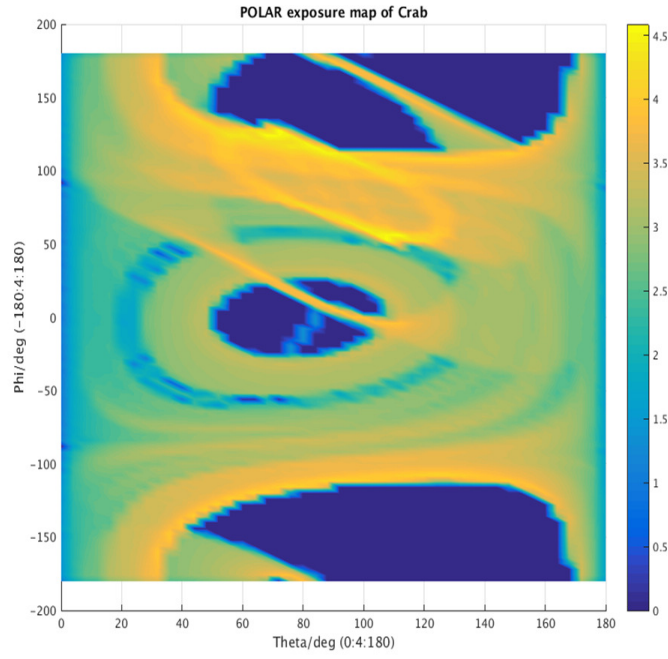

(b)

Figure 2: (a) is the structure of POLAR detector. It consists of 25 modules, and each module has $8 * 8$ detecting channels made of plastic scintillator bars. The incident angles are defined as theta and phi. Theta angle is the angle of $\mathrm{Z}$-axis positive direction and the source vector from origin of coordinates to source point. Projecting the source vector onto $\mathrm{X}-\mathrm{Y}$ plane, and phi is the angle between projection and $\mathrm{X}$ axis positive direction. (b) is the Crab exposure map on POLAR. It does not cover all incident directions. 


\subsection{POLAR data reduction}

Crab photons are drown in a sea of background. Data reduction is necessary to reduce background. First, the acquisited data lack of PPD should be rejected, since we are not clear the incident angle without PPD, and we don't know whether there is crab photon in SCI. Second, in order to ensure that crab is within our visible region, the event whose theta incident angle larger than 100 degrees should be rejected. At last, in view of the scattering times of Crab photons in POLAR detector rarely more than 5 , we throw away the event whose trigger number more than 5 . The above reduction methods can improve ratio of signal and noise for Crab analysis.

\subsection{Timing process}

We study the timing properties of the Crab pulsars with the POLAR observations. First, the arrival time for each photon was converted to the Solar System Barycentre using ephemeris DE405. For each observation we obtained the period of a pulsar by folding the observed counts to reach the maximum Pearson $\chi^{2}$. Second, the pulse profile was folded with the spin frequency and the time of arrival (TOA) was calculated from the peak phase of the pulse and the reference time. Then timing parameters were solved by the phase coherent timing method utilizing TEMPO2 [14] [15]. At the end, the Fermi-LAT ${ }^{2}$ observations were analysed with the same process to check whether the observation by POLAR was performed appropriately especially for the timing system.

\section{Results from POLAR}

\subsection{Timing results}

From the almost consecutive observation from POLAR, we have obtained the frequency evolution and timing results as shown in Figure 3 and Table 1. First of all, the spin frequency of the Crab pulsar is checked. Due to the large background for the Crab pulsar, we combined the observation data in every day to search the spin frequency of the pulsar. As shown in Figure 3 (a), the spin frequency of the pulsar decreases with time significantly with time, which is consistent with the ephemeris supplied by Jodrell Bank [16] ${ }^{3}$. Then, TOAs were calculated with the spin parameters and fitted utilizing TEMPO2. With the best parameters, the timing residuals distribute near zeros with the root mean squared value $85 \mu$ s. However, the timing residuals show slow variations with time as the Crab pulsar has the large timing noise as shown in Figure 3 (b). In order to verify these results, we also checked timing residuals observed from Fermi-LAT the at the same time interval with the same spin parameters. As illustrated in Figure 3 (b), the timing residuals observed by POLAR are remarkably consistent with Fermi-LAT results, which suggests that timing system of POLAR is reliable.

\subsection{Pulse profile}

With the accurate timing parameters, the total pulse profile of the Crab pulsar was folded from the all observed events. As shown in Figure 4 (a), the pulse profile shows the typical double-peak

\footnotetext{
${ }^{2}$ https://fermi.gsfc.nasa.gov/ssc/data/analysis/LAT_essentials.html

${ }^{3}$ http://www.jb.man.ac.uk/pulsar/crab.html
} 
Table 1: The timing parameters of the Crab pulsar

\begin{tabular}{cc}
\hline \hline Parameters & Value \\
\hline \hline PEPOCH(MJD) & 57697.040344079745 \\
F0(Hz) & $29.6484272934(4)$ \\
F1 $\left(10^{-10} \mathrm{~Hz} \mathrm{~s}^{-1}\right)$ & $-3.689865(1)$ \\
F2 $\left(10^{-20} \mathrm{~Hz} \mathrm{~s}^{-2}\right)$ & $1.16(1)$ \\
F3 $\left(10^{-28} \mathrm{~Hz} \mathrm{~s}^{-3}\right)$ & $3.4(3)$ \\
\hline
\end{tabular}

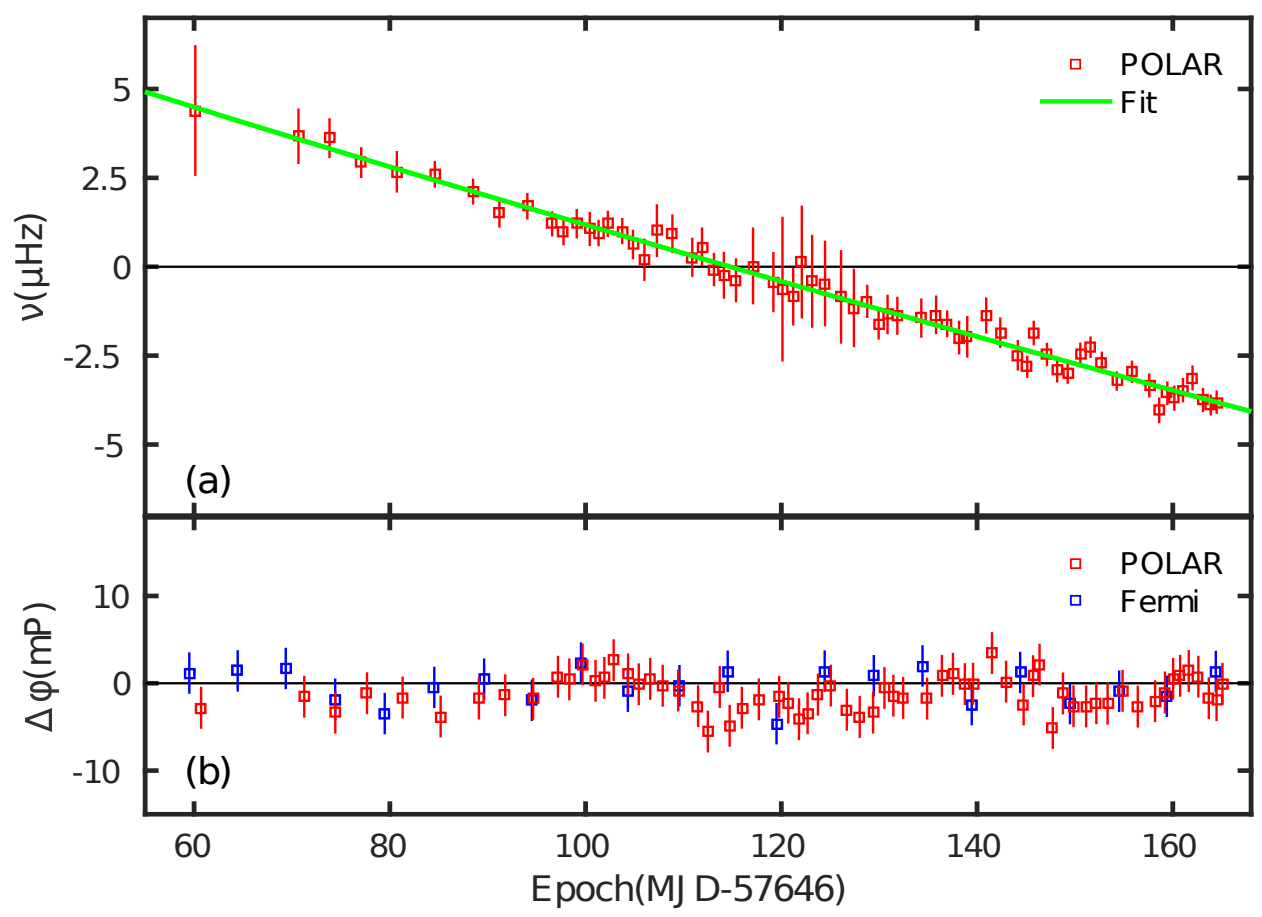

Figure 3: Panel (a): the evolution of the spin frequency of the Crab pulsar observed by POLAR. Each data point is subtracted by $\left(29.648422-t * 3.68 \times 10^{-10}\right)$ to show the details of its frequency evolution. The green line represents the fitted result. Panel (b): The time residuals of the Crab pulsar observed by Fermi and POLAR, as represented by the blue and red squares respectively.

structure with high significance, which is also consistent with the results of RXTE [17]. As the large of view of POLAR, Crab could be observed in every orbit and pulse profile could be obtained though with lower significance. Therefore, all pulse profiles observed in every day are co-aligned with the same phase as illustrated in Figure 4 (b). These results also confirm that POLAR has detected the pulsed photons from the compact objects.

The pulse profile as a function of theta incident angle as shown in Figure 5 (a), which indicate that POLAR's FoV in its energy range is larger than 100 degrees. And the pulse profile as a function of 1600 channels as shown in Figure 5 (b), which show that the pulsed photons of Crab are captured 


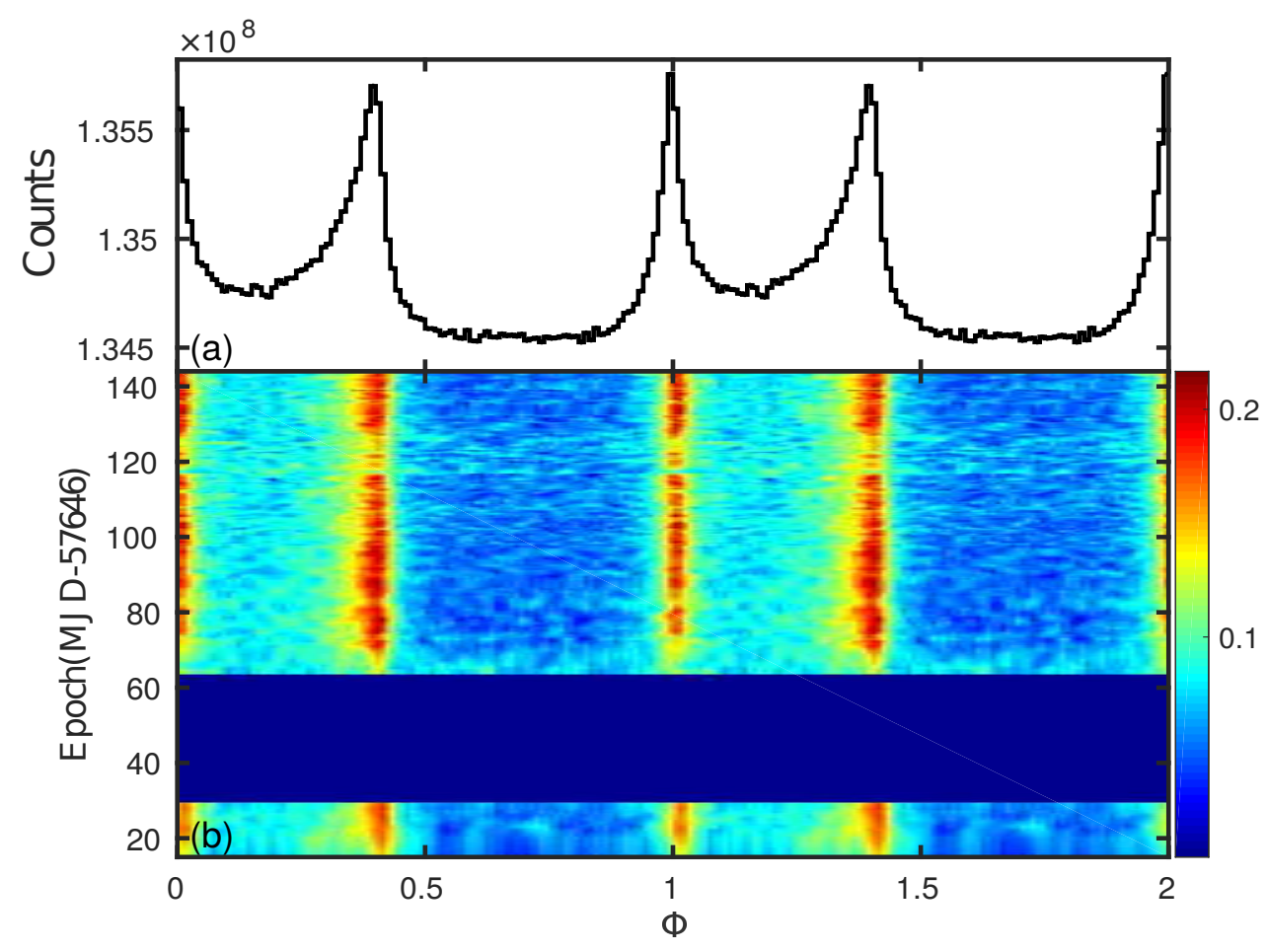

Figure 4: The pulse profile detected by POLAR as function of time. Panel (a) shows the total pulse profile accumulated from all observations. Panel (b) exhibits the normalized profiles with background subtraction observed in every day.

by every channel.

\subsection{Potential polarization measurement}

All information of an event with its scattered photon(simultaneous trigger) is recorded by detector. Generally, the first reaction point has maximum deposited energy, and the second reaction point takes second place. Projecting the direction from the first reaction point to the second reaction point onto $\mathrm{X}-\mathrm{Y}$ plane, the angle between projection and $\mathrm{X}$-axis is the azimuthal angle of this compton scattering event. For GRB, since it's short duration, accumulating the events in different azimuthal angle, we obtain modulation curve to reveal polarization information. However, for Crab pulsar the incident angle between the Crab and POLAR varies with time, so we have to obtain modulation curve as a function of incident angle. Then, we use POLAR Monte Carlo simulation software package [18] to reappear Crab observation on POLAR. This software package was developed from a calibration experiment on POLAR at European Synchrotron Radiation Facility. Simulating different polarization Crab source in parallel, and comparing these simulation results with observation results. If simulation results of one presuppose polarization are consistent with observation results, then we consider this presuppose polarization as measured polarization of Crab pulsar.

However, the precondition is that we should use Monte Carlo simulation to reappear the ob- 


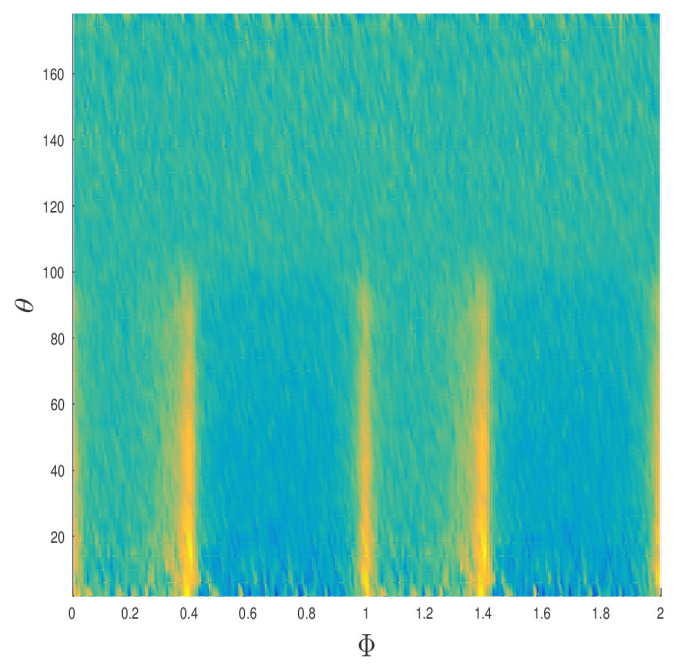

(a)

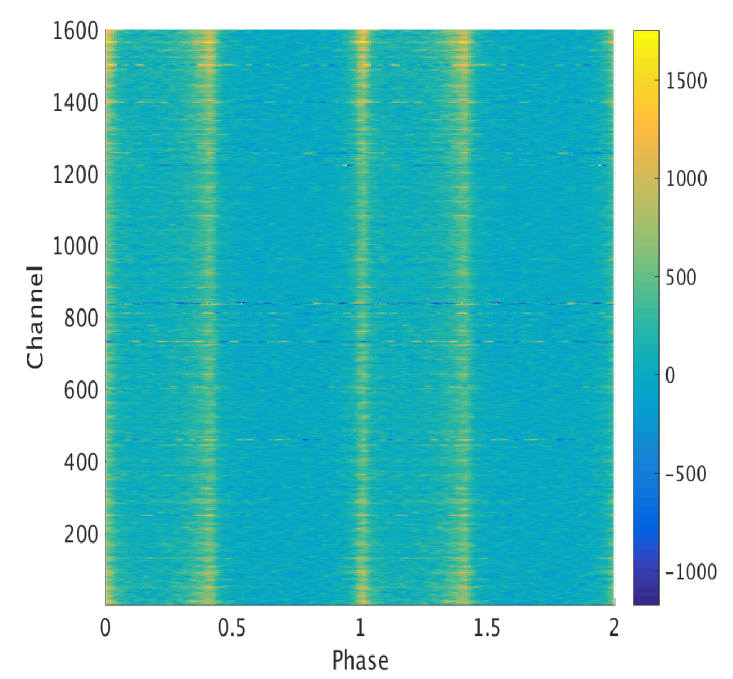

(b)

Figure 5: (a) is Pulse profile as function of theta; (b) is pulse profile for 1600 channels.

servation results. In other words, we need a caibration process. The response of Crab detection varies with many factors, incuding but not limited to detection efficiency, projected area, visible efficiency, cnts Rate and so on. Look for as many factors as possible from observation-simulation conjoint analysis, and make them decouple with polarization. Then polarization measurement is the potential targert.

\section{Summary}

Firstly, from the observations, POLAR detected highly significant pulsed signals from the Crab pulsar which is similar with the known results. The pulse profile as a function of theta incident angle indicate that POLAR's FoV larger than 200 degrees, and as a function of 1600 channels shows crab photons captured by every channel. Second, the accurate timing behavior observed by POLAR is highly consistent with Fermi-LAT observation, it verified POLAR timing system with the stable clock $85 \mu \mathrm{s}$. The above results show that POLAR has a good detection performance. And lastly, we have potential to measure the polarization of Crab on POLAR.

\section{Acknowledgments}

We thank the High Energy Astrophysics Science Archive Research Center(HEASARC) at NASA/Goddard Space Flight Center for maintaining its online archive service that provided the data used in this research. This work is supported by the National Key Research and Development Program of China (2016YFA0400802), National Science Foundation of China (11233001, 11503027, 11303069 and 11503028), and the Strategic Priority Research Program on Space Science, the Chinese Academy of Sciences,Grant No. XDA04010300 and XDB23000000. 


\section{References}

[1] Eikenberry, S. S., and Fazio, G. G. 1997, ApJ, 476, 281

[2] Kuiper, L., Hermsen, W., Cusumano, G., et al. 2001, A\&A, 378, 918

[3] Rots, A. H., Jahoda, K., Macomb, D. J., Kawai, N., Saito, et al. 1998, ApJ, 501, 749

[4] Molkov, S., Jourdain, E., and Roques, J. P. 2010, ApJ, 708, 403

[5] Ge, M. Y., Lu, F. J., Qu, J. L., Zheng, S. J., Chen, Y. and Han, D. W. 2012, ApJS, 199, 32

[6] Novick R., et al., 1972, ApJ, 174, L1

[7] Weisskopf M. C., et al., 1976, ApJ, 208, 125

[8] M. Forot, et al., Astrophys. J. 688 (2008) L29

[9] A.J. Dean, et al., Science 321 (2008) 1183.

[10] M. Chauvin, et al. 2017, arXiv:1706.09203 [astro-ph.HE].

[11] M. Kole et al., ICRC Conf. Proc. 2017

[12] Sun, J. C., Doctoral Thesis, UCAS 2012, at http://ir.ihep.ac.cn/handle/311005/210194

[13] N. Produit et al., Nucl. Instr. and Meth. A 550 (2005) 616.

[14] Edwards, R. T., Hobbs, G. B., \& Manchester, R. N. 2006, MNRAS, 372, 1549

[15] Hobbs, G. B., Edwards, R. T., \& Manchester, R. N. 2006, MNRAS, 369, 655

[16] Lyne, A. G., Pritchard, R. S. and Graham-Smith, F. 1993. MNRAS, 265, 1003

[17] Ge, M. Y., Yan, L. L., Lu, F. J., et al. 2016, ApJ, 818,48

[18] M. Kole, Li,Z. H., et al., submitted to Nucl. Instr. and Meth. A 